\title{
The impact of oxidative stress in androgenic alopecia in women
}

\author{
Anna Cwynar', Dorota M. Olszewska-Stonina ${ }^{1}$, Rafał Czajkowski²
}

1Department of Pathobiochemistry and Clinical Chemistry, Collegium Medicum in Bydgoszcz, Nicolaus Copernicus University in Torun, Poland

2Department of Dermatology, Sexually Transmitted Disorders and Immunodermatology, Collegium Medicum in Bydgoszcz, Nicolaus Copernicus University in Torun, Poland

Adv Dermatol Allergol 2020; XXXVII (1): 119-120

DOI: https://doi.org/10.5114/ada.2019.81685

Androgenic alopecia (AGA) is one of the most common forms of female pattern baldness, and the incidence of this disease entity increases with age. It is estimated that AGA occurs in about $10 \%$ of women in the pre-menopausal period and in 50-75\% after the age of 65 [1].

The diagnosis of AGA in women is difficult. No reference diagnostic method has been developed so far which would be helpful both in diagnosis and monitoring of treatment of this form of alopecia. One of the basic examinations is the trichological analysis which allows to assess the percentage of hair at particular stages of growth [2]. Another parameter helpful in the diagnosis of AGA is the determination of the level of hormones in the blood - free and total testosterone, dehydroepiandrosterone and oestrogens. However, the androgen levels in AGA patients may be increased or normal [1].

In androgenic alopecia, dihydrotestosterone (DHT) stimulates the secretion of hair growth inhibitory factors, such as the transforming growth factor $\beta 1$ and 2 (TGF- $\beta 1 / p 2$ ) [3]. The study by Upton et al. has shown that in vitro culture of dermal papilla (DP) cells, derived from the scalp of male patients with AGA, undergoes premature aging. The main cause of this process is oxidative stress and the gradual accumulation of reactive oxygen species (ROS) in cells, resulting in the loss of their function. ROS mediate in response to DHT and growth factors, which in turn may affect growth factor signalling in the cell [3].

This study aims to investigate oxidative stress in female patients with AGA and in healthy controls by measuring the levels of plasma and erythrocyte malondialdehyde (MDA), the level of the ceruloplasmin (CER) and the activity of paraoxonase 1 (PON1). The study included 12 AGA female patients (mean age: $46.8 \pm 13$ ) and the control group consisting of 25 age- and sex-matched healthy volunteers (mean age: $32.7 \pm 11$ ). There was no significant body mass index (BMI) difference between groups.

Plasma and erythrocyte MDA levels were higher in female patients with AGA compared with controls (by $4.76 \%$ and $10.99 \%$, respectively), but these differences were not statistically significant ( $p=0.205 ; p=0.909$ ). However, a significant increase was observed in CER levels $(p=0.015)$ in the studied group compared with the controls. In contrast, the activity of PON1 was significantly reduced $(p=0.038)$ in the studied group compared with the healthy volunteers (Table 1 ).

The skin is constantly exposed to both endogenous and exogenous oxidizing agents. The action of ROS results in damage to cellular components, such as nucleic acids, proteins and lipids of the cell membrane. The results obtained indicate a disturbance of the oxidativeantioxidant balance in the course of AGA in women.

Lipid peroxides and their products (MDA) may cause disturbances in the normal functioning of most cells and their level correlates with the degree of lipid peroxidation $[4,5]$. The increase in lipid peroxidation products in female patients with AGA suggests the presence of oxidative stress.

Ceruloplasmin belongs to preventive antioxidants, and its ability to bind transition metal ions prevents the formation of superoxide anion radical $\left(\mathrm{O}_{2}{ }^{-}\right)$[6]. CER is attributed to approximately $80 \%$ of plasma antioxidant properties [7]. An increased concentration of this enzyme in blood plasma, observed in the course of inflammation, is most likely associated with the body response to the oxidative stress accompanying these processes [8]. The observed increase in the CER concentration in female patients with AGA may support the hypothesis of androgenetic alopecia as an inflammatory disease.

Paraoxonase 1 is an enzyme involved in the hydrolysis of ester bonds in the body. Increasing interest in

Address for correspondence: Anna Cwynar MD, PhD, Department of Pathobiochemistry and Clinical Chemistry, Collegium Medicum, Nicolaus Copernicus University, 9 M. Curie Skłodowskiej St, 85-094 Bydgoszcz, Poland, phone: +48 793414790 , e-mail: anna.cwynar@cm.umk.pl Received: 8.08.2018, accepted: 1.10.2018. 
Table 1. The values of MDA, CER and PON1 in peripheral blood of androgenic alopecia female patients and healthy subjects

\begin{tabular}{lccc}
\hline Parameter & $\begin{array}{c}\text { AGA patients } \\
(n=12) \\
\text { Me }(\mathbf{Q} 25-\mathbf{Q} 75)\end{array}$ & $\begin{array}{c}\text { Healthy subjects } \\
(n=25) \\
\text { Me (Q25-Q75) }\end{array}$ & $P$-value \\
\hline MDAe [nM/gHb] & $22.22(18.37-29.71)$ & $20.02(15.86-24.10)$ & 0.909 \\
\hline MDA [nM/ml plasma] & $0.44(0.42-0.49)$ & $0.42(0.38-0.46)$ & 0.205 \\
\hline CER [mg/dl] & $27.35(24.65-32.75)$ & $23.09(20.50-25.20)$ & 0.015 \\
\hline PON1 [U/l] & $39.04(29.16-50.52)$ & $50.75(38.12-81.06)$ & 0.038 \\
\hline
\end{tabular}

AGA - androgenic alopecia, Me - median, MDAe - malondialdehyde concentration in erythrocytes, MDA - malondialdehyde concentration in blood plasma, CER - ceruloplasmin, PON1-paraoxonase 1, $p$-statistical significance level (Mann-Whitney U test).

this molecule, however, is primarily associated with its antioxidant activity. PON1 takes part in the degradation of hydrogen peroxides and the metabolism of xenobiotic substances, playing an important role in the body detoxification process $[9,10]$. The observed reduced activity of the PON1 enzyme in the studied group's serum may play a role in the progression and/or development of AGA. Further research is needed to clarify these results.

There are still few literature reports investigating the oxidative stress parameters in the blood of female patients with androgenetic alopecia. Therefore, it was considered interesting to study the antioxidant systems in erythrocytes and plasma of this group of patients.

\section{Acknowledgments}

The authors thank the Department of Medical Biology (Nicolaus Copernicus University in Torun) for technical support during the preparation of this manuscript.

\section{Conflict of interest}

The authors declare no conflict of interest.

\section{References}

1. Urysiak-Czubatka I, Broniarczyk-Dyła G. Examination of hair growth parameters in androgenetic alopecia in women using TrichoScan. Post Dermatol Alergol 2010; 27: 246-56.

2. Moghadam-Kia S, Franks AG. Autoimmune disease and hair loss. Dermatol Clin 2013; 31: 75-91.

3. Upton JH, Hannen RF, Bahta AW, et al. Oxidative stressassociated senescence in dermal papilla cells of men with androgenetic alopecia. J Invest Dermatol 2015; 135: 1244-52.

4. Hauck AK, Bernlohr DA. Oxidative stress and lipotoxicity. J Lipid Res 2016; 57: 1976-86.

5. Briganti S, Picardo M. Antioxidant activity, lipid peroxidation and skin diseases. What's new. J Eur Acad Dermatol Venereol 2003; 17: 663-9.

6. Wołonciej M, Milewska E, Roszkowska-Jakimiec W. Trace elements as an activator of antioxidant enzymes. Postepy Hig Med Dosw 2016; 70: 1483-98.

7. Bartosz G. Druga twarz tlenu - wolne rodniki w przyrodzie. Wyd. PWN, Warsaw 2013.

8. Lee MJ, Jung CH, Kang YM, et al. Serum ceruloplasmin level as a predictor for the progression of diabetic nephropathy in
Korean men with type 2 diabetes mellitus. Diabetes Metab J 2015; 39: 230-9.

9. Zielaskowska J, Olszewska-Stoniba D. The polymorphism of paraoxonase and its effects in physiological and pathological processes. Adv Clin Exp Med 2006; 15: 1073-8.

10. Łukaszewicz-Hussain A. Paraoxonase activity and lipid peroxides concentration in serum of rats subchronically intoxicated with chlorpyrifos - organophosphate insecticide. Medycyna Pracy 2012; 63: 559-64. 